\title{
MISUSE OF DERIVATIVES: CONSIDERATIONS FOR INTERNAL CONTROL
}

\author{
Simon Grima, Frank Bezzina and Inna Romānova
}

\begin{abstract}
Derivatives are nowadays widely used globally both for speculative and hedging purposes. However, as experience shows, inadequate use of derivatives may cause severe problems and even bankruptcy of firms. Thus, it is essential to help organizations design a robust proactive governance and internal control structure, which will help to prevent new financial debacles and scandals when using derivatives. Taking into account the frequent use and the growing fraud caused by derivatives, the aim of the paper is to identify considerations for internal control important to ensure better governance of firms using derivatives. The main findings are based on an analysis of interviews that were conducted with experts directly or indirectly involved with derivatives from different European countries. The interviews were semistructured following the approach proposed by Patton (1990). An analysis of the data collected from the interviews was carried out using a thematic approach. The paper identifies and analyzes the main "sources" of derivatives misuse, including poor design and mis-categorization of instruments, convenience to blame
\end{abstract}

Contemporary Issues in Finance: Current Challenges from Across Europe

Contemporary Studies in Economic and Financial Analysis, Volume 98, 49-62

Copyright (C) 2017 by Emerald Group Publishing Limited

All rights of reproduction in any form reserved

ISSN: 1569-3759/doi:10.1108/S1569-375920160000098004 
derivatives, unsophisticated players, insufficient regulatory environment, poorly designed internal controls, inadequate communication, poor firm culture, etc. It also provides an extensive analysis of the main recommendation for internal control concerning awareness of derivatives design, the human aspects, regulations, communication, knowledge, and training. Sound internal controls could avoid new debacles without adding other restrictions to the market. Moreover, it provides recommendations for internal control important to ensure better governance of firms using derivatives.

Keywords: Derivative misuse; internal control; communication; firm culture; regulatory environment

JEL classifications: G23; M42

\section{INTRODUCTION}

Activity in derivatives is expanding rapidly, globally and has been blamed for most of the devastating financial disasters. The debate on their control through good governance and regulation is still somewhat unresolved. Not a rare occurrence when using derivatives is misuse, as well as lack of focus on those risk management areas, those compliance areas, those settlement areas, that can ultimately save money. "However, without undervaluing the importance of regulation and the usefulness of an adequate external control, a sound internal control system is essential to prevent new financial scandals" (Fernández-Laviada, Martínez-García, \& Montoya Del Corte, 2007).

In July, 1993, the Group of Thirty, Washington, DC - Global Derivatives Study Group, published "Derivatives: Practices and Principles," with a scope of developing appropriate practices for proper derivative use. They concluded that derivatives by their nature are not any more risky than other financial instruments and therefore can be addressed within the current structures and practices. The key focus should therefore be on clarifying regulatory and standards inconsistencies that might impede riskreduction. In response to the derivatives-related problems over the years, many of which are said to have resulted from the misunderstanding of risks and their use for risk management purposes, the Committee of Sponsoring Organizations of the Treadway Commission issued "the COSO report, 
Internal Control-Integrated Framework in 1992," as a guidance for Internal Control Issues in Derivatives Usage (1992). Herein, they have given importance to the formulation of policies governing derivatives use for risk management. That is to help ensure that management directives are carried out with an understanding of the environment they are in, the human aspect (integrity, ethical values, and competence of the entity's personnel, as well as management's philosophy and operating style, oversight, monitoring, and assessment to reach objectives), knowledge of frameworks, models, and tools available and their assumptions and limitations, information and communication (the nature and quality of information needed, the systems used to collect such and reports necessary). This report stresses the importance of communications and that it should be such that ensures "that duties and control responsibilities relating to derivative activities are understood across the organization" (COSO, 1992).

According to Bezzina and Grima (2012) attention is needed when using derivatives to be given to areas such as risk management, human greed, politics, inappropriate standards, and inadequate controls. They noted that users and controllers are capable of dealing with derivatives even in complex situations; derivatives are valuable financial instruments; and they are aware of the benefits derivatives provide to firms, when properly handled. They also highlighted the importance given by the respondents to education, position held, and experience with derivatives (Bezzina \& Grima, 2012).

Internal controls were also examined by Géczy, Minton, and Schrand (2007), who noted the need for proactive frameworks which firms can tailor to monitor derivatives use. Zeidan and Müllner (2015) reveal that some corporate governance characteristics are relevant to the mismanagement of derivatives instruments. Allayannis, Lel, and Miller (2012) found that wellgoverned firms are more likely to use derivatives to hedge rather than to speculate or pursue managers' self-interest. Fernández-Laviada et al. (2007) revealed that main concern related to the lack of experience and adequate knowledge of derivatives and noted that there is, however, sufficient control and attribute losses to adverse market movements. They found that the relationship between firms suffering losses and those speculating in derivatives to be positive.

\section{Aim of the Paper}

The belief that "derivatives don't kill companies - people using derivatives kill companies!" noted by Barbara Davison (2000) in the opening sentences 
to her book "Auditing Derivative Strategies" and the above works motivated the authors to understand better the firm's governance and internal controls and to determine the considerations to take into account when designing a structure that can ensure the positive use of derivatives. This is essential to help organizations design a robust proactive governance and internal control structure, which will help to prevent new financial debacles and scandals when using this instrument or help to ensure that the truth about these scandals is revealed and not blamed on derivatives. Besides, internal controls are important to a wide range of stakeholders also outside the firm as investors, government, and society as a whole (Kinney, Maher, \& Wright, 1990).

Taking into account frequent use and the growing fraud caused by derivatives, this paper aims to identify recommendations for internal control, important to ensure better governance of firms using derivatives.

\section{METHODOLOGY}

In this study the authors chose to use interviews with 86 persons directly or indirectly involved with derivatives (chosen from within the United Kingdom, Italy, Switzerland, Czech Republic, Germany, Latvia, and France), as a tool to understand and explain the problem. The authors ensured that the interviewees represented a wide range of participants and perspectives on derivative use. They did this by using a nonprobability purposeful sampling, since to be relevant for this study the participants chosen had to have sufficient years of experience and knowledge in the field of derivative use or control. Besides, they also identified and interviewed some persons who they knew passed through a rough time with their firms because of derivative misuse.

The interviews were semistructured, with the interviewer asking four questions, one to determine demographics, that is, the "experience," "academic background," and "position held within the firm"; and three open-ended questions to determine (a) whether the company has ever been in difficulty because of derivatives, (b) whether they believe that derivatives are bad instruments and are rightly blamed for firm debacles, and (c) what are the considerations one should take when dealing with derivatives so as to ensure proper use; but allowing the interviewees the freedom to elaborate and open up as much as they desired. This enabled the authors to capture experience/information/ideas/opinions which otherwise could not 
have been obtained and could have been lost. The style of the interview followed was that proposed by Patton (1990) - a general interview guide approach in contrast with the informal conversational interview and the standardized open-ended interview.

While the general topic of derivatives use is common in all the interviews in the study, the researchers constructed specific questions, which seemed appropriate for interviewees. The guides allowed the researchers to be flexible and responsive to unexpected paths and discoveries during the interview. Patton (1990) explains that the interviewer remains free in building a conversation within a particular subject area, to word questions spontaneously, and to establish a conversational style, but with the focus on a particular subject that has been predetermined.

The order of questions may also have been varied depending on the flow of conversation and, given the nature of events within the particular division, additional questions may have been required to explore further. Moreover, the nature of the questions and the ensuing discussion means that the data are recorded, by note-taking. Accordingly, the semistructured interviews provides the researchers with the opportunity to "probe" answers, which can be done in instances where there is a need or want for the interviewees to explain further or build on their responses (Saunders, Lewis, \& Thornhill, 2003).

During the interview, the interviewers were able to encourage the interviewee to clarify vague statements or to elaborate further on brief comments. The interviewers maintained objectivity and did not attempt, in any way, to influence the interviewee's statements.

The researchers visited various derivative forums, joined associations, and followed and intervened in various derivative-related group discussions such as LinkedIn, AuditNet, Global Derivatives, and Wilmott to obtain contacts/candidates for interviews. The various tools used to carry out the interviews included MSN, Skype, Google talk, Viber, and WhatsApp, various derivatives forums, face-to-face interviews, and telephone/mobile. The authors' experience and knowledge enabled to join the above discussions and also to participate in some webinars and surveys. By this, they gained the trust of candidates who then eagerly participated in the interviews.

An analysis of the data collected from the interviews was carried out using a thematic approach (Braun \& Clarke, 2006). The authors did this manually and in (4) steps: preparation of manuscripts of interviews; identification and assignation of codes, that is, a letter referring to the descriptive and interpretative codes: (a) Poor design and mis-categorization of the 
instrument, (b) Convenience, (c) Unsophisticated players, (d) Regulatory environment, (e) Complex instruments, (f) Poorly designed internal controls, (g) Communication, (h) Complacency, and (i) Political pressure next to the relevant text on these transcripts. The themes of the interviews were finally drawn-up and given names (as noted above) from these codes and a descriptive final report as well as summary of the information and analysis was then compiled.

The technique used here relies on the respondent's willingness to give accurate and complete answers (Breakwell, Hammond, and Fife-Schaw, 1994). For a variety of reasons, they may refrain from telling the truth (e.g., due to feelings of embarrassment, inadequacy, bluff about knowledge on the topic, nervousness, memory loss, or confusion). The authors used their experience, online forum discussions, and peers to ensure that the codes/themes were chosen and analyzed correctly. Moreover, after completing the first review, the researchers left some time and went back to review them again to ensure they did not miss out or misinterpret anything.

According to Guest, Bunce, and Johnson (2006) "guidelines for determining non-probabilistic sample sizes are virtually non-existent. Purposive samples are the most commonly used form of non-probabilistic sampling, and their size typically relies on the concept of 'saturation', or the point at which no new information or themes are observed in the data." The authors felt that this point had arrived well before the amount of participants interviewed (i.e., at 63 interviews).

Notwithstanding that, the authors strived hard to convince females to participate in the interview, by contributing and participating in a group studying "women in financial services" on LinkedIn, the majority of those contributing to the interview were male (65).

With respect to position held, the participants were grouped into two categories for ease of comparison - users and controllers of derivatives. The largest number of participants in the interview (63) was controllers, who were in the main, Internal Auditors (41), Risk Managers (11), Regulators (3), and Compliance Managers (8). The other category has all been/is involved in trading derivatives.

Most of the participants (74) have between 11 and 20 years' experience or more in the use or control of derivatives. The rest had less than 11 years' experience but more than 8 years. This is consistent with the aim of the authors to interview persons with vast experience and knowledge on the subject.

The distribution of those interviewed is skewed towards those holding postgraduate education (48) showing that most participants hold a 
Master's degree or a higher level of education. Only (12) participants hold a diploma. This shows the importance given to education by users and controllers of derivatives.

\section{RESEARCH RESULTS AND DISCUSSION}

To set internal controls, one needs to understand the subject in question and what the contributors to derivative misuse are. Based on the analysis of interviews the authors have identified the main "sources" of derivatives misuse (see Fig. 1).

Poorly designed and mis-categorized instruments - A total of (54) of the participants in the interview showed concern about the fact that some forms of derivatives, such as for example, credit default swaps, were "poorly designed" and "mis-categorized," allowing for excessive risk (credit risk) being taken on the part of the sellers. Moreover, they explained that these instruments in their opinion are more insurance vehicles than derivatives and that they have more to do with human design than with the instrument itself. However, slightly more than half of the participants (46) noted that derivatives create dangers and risks as do other instruments, but people facilitate their misuse. Therefore, in their opinion, the amount and quality of risk taken is a human choice, derivatives only enable and give this choice.

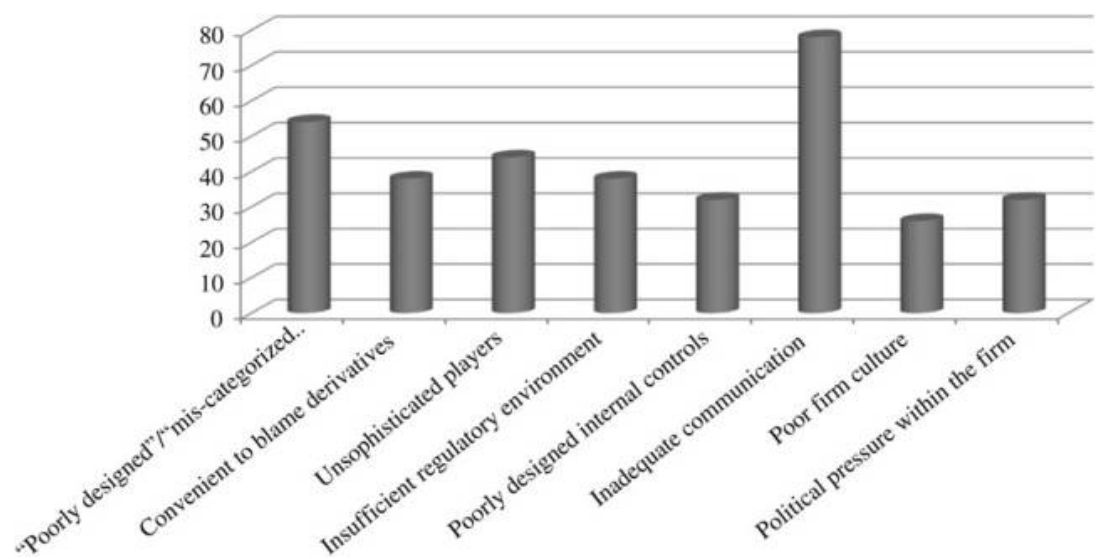

Fig. 1. The Main Contributors to Derivative Misuse. Source: Authors' construction based on the interviews. 
Convenience - A total of (38) noted that one must be aware of the noise around derivative misuse. They mention that people find it is convenient to blame derivatives for financial disasters or scandals. Therefore, it is important that the controls do not stop the use of derivatives, since these may be the only most effective instruments that one can use to manage risks and therefore, set the most efficient internal controls.

Unsophisticated players - A total of (44) have mentioned that the evolution and growth of the risk management profession will ensure that derivative products will continue to exist and evolve. They were however unsure on the extent and types of derivatives. The enhanced presence of the internet and globalization was mentioned by them as one of the tools, which will speed up derivative transactions and open them up to a wider population. However, there were concerns that this would lead to problems because of more unsophisticated players who will be able to enter the market. Also, the larger the firms and the more complex its capital structure is, the more is the requirement for customized risk management instruments. The risk being that the policies in place and the controls are not adequate to ensure appropriate use of these derivatives. Even so, because of the fact that innovations run ahead of controls and due to the unfortunate fact that firms' culture is more often one that looks at controls as a cost.

Regulatory environment - A total of (38) mentioned that changes in the next few years would be driven largely by the regulatory environment, creating the need for new products or change in existing products. They noted current changes in, and forthcoming regulations, standards, and recommendations will undoubtedly inspire innovation and that one should not be surprised to see firms come up with products designed to work around them. "Ultimately, to enforce the Basel recommendations properly or any other regulations and standards will take some time and by this time derivatives would have evolved and mushroomed" (Grima, 2011). Moreover, they noted that these have not yet completely managed to address the problems and risks brought on by derivatives and although they can help increase awareness and put in some structure and methodologies to help account and report them, they are not a solution to the problem. They continued explaining that one should be aware of the false illusion that by addressing these requirements their companies are safe. If they are inappropriately drawn-up, they can increase the risks.

They mentioned that all market players and controllers should participate in consultation forums to drive change where these are inappropriate and mentioned that the risk associated to certain positions was not being 
calculated correctly because information was not always available and complete and the methodology/models used to assess risk did not function correctly nor had too many assumptions. Therefore, this makes any capital requirement regulations worthless. Moreover, some transactions are so complex that they are understood only by their creator, sometimes using complicated mathematics that even the biggest "Gurus" cannot understand. Whether their calculations are correct, whether they are hedging or speculating, or whatever the risk they are facing, will only be revealed in the case of a large loss.

Poorly designed internal controls - A total of (32) participants noted that in each of the debacle episodes blamed on misuse of derivatives, the fault has lain with one "Rogue Trader" who has circumvented the rules and assumed excessive trading positions that have resulted in substantial financial losses or bankruptcies (this relates also to the convenience consideration mentioned above). This presented a problem that whilst certainly not systemic indicated that the "internal controls" were poor. It was also highlighted that although derivatives had been increasing dramatically, changes in controls and governance procedures were only few. They explain that external audits will never be sufficient; these need to be supplemented with a good, efficient, powerful internal audit function.

Communication - A total of (78) participants highlighted that the problem was that controllers and users see each other as enemies with opposite goals: front-office personnel are expected to make money whatever the risk involved to ensure target meeting and controllers who are often seen as obstacles and value wasters. In their opinion communication between the parties needs to be strengthened. Users, as they explained, are the first line of defense for a firm and without their help the controllers will not be able to draw-up the best controls possible for the company. The sentiment of these participants is that consistent with the statement by Kang (2007), "the first line of defence is always at home. Knowing the ingredients and recipe of what's being cooked (or more importantly, what you're eating) is in your best interest." The culture of firms should be changed to one where it is accepted by the controllers, that management is the first line of internal control/defense of a firm. They are the ones who have the most knowledge of what is going on and are the real controllers. However, current environment and culture, in their opinion, drives users and controllers apart. Also, the frequency with which the Board of Directors and Senior Management are informed of the risks and trade positions taken, as well as of any other information necessary for taking appropriate decisions is not always 
forthcoming and timely. Besides, the participants highlighted that communication flow and structure in internal controls was not always adequate, mainly because the persons heading the areas were not knowledgeable enough or did not have the appropriate experience and character.

Complacency - A total of (26) participants highlighted complacency, as the core reason in each and every financial disaster. This, they believe, primarily occurs because of the fundamental attribution error that, "it won't happen to us, it will happen to somebody else." This can make controllers become complacent with their work and keep them from highlighting problems.

Political pressure - A total of (32) participants noted that political pressure exists within firms and have a part to play in derivative use, but they believe it to be more subconscious than conscious - that is, the more profit-making sections of the organization exist on a pedestal. In some cases (15) political pressure was blended with greed, hubris, and the desire for power. In this respect they compare the use of derivatives as an investment tool to glorified gambling in the wrong hands.

\section{Considerations for Internal Control Design}

The analysis of these interviews helped the authors reveal the main "sources" of derivatives misuse. Adequate control and appropriate governance may help to completely avoid or substantially decrease the risk of derivatives misuse.

Often innovations run ahead of controls, therefore, to ensure appropriate use of derivatives, controllers should ideally have as much knowledge as the trader, on the instrument and should not be put in a position of fear to ask questions. Controls and derivative use would ideally run in parallel and guidelines drawn-up for innovations. The controllers need to be knowledgeable, innovative, imaginative, and flexible enough to create the best solutions and polices to cater for these kinds of derivatives. Controllers should be aware of the considerations identified herein and determine and understand the knowledge and education that market players are required to have, set minimum standards on these, and ensure that they are continually updated and trained.

It is important to have strong Board and committee (Audit, Compliance, and Risk Management) members, which meet regularly and keep an open communication flow with the internal control units. The issue is that these are not always made up of people experienced in control areas 
and many times are posts filled by persons who see it as a resting/retirement position.

In order to ensure appropriate firm culture and avoid opposite activities of controllers and users, seeing each other as enemies, communication between the parties needs to be improved. To ensure this, in some firms the internal control design is seen as a project that involves all stakeholders within the firm: representatives from different departments and management levels, including front-office and back-office. This approach enables more effective communication as well as allows closing the "knowledge gap" of some participants. Moreover, it ensures later better understanding and acceptance of the internal control functions.

A total of (57) participants stated that they are doing well with derivatives and noted that this was due to recruiting the appropriate staff members, good communication, and controls in place. They explained that the process for managing derivative trades safely is generally something that evolves as a consequence of experience. They noted that usually firms adjust their policies and practices incrementally, to incorporate perceived improvements. The best solution is to have controls and usage of derivatives growing together at the same speed. These participants have indicated their control structure and key control indicators as the secret to successfully safeguarding against derivative misuse and have indicated a system of integrated internal control units (with some variations). They believe that globalization, the evolution of capital markets and macroeconomic changes are increasing the challenges and creating unprecedented opportunities for companies. Today, many firms understand the importance of linking the internal control departments, ensuring governance, and the need for good communication between the so-called "three lines of defense." They note that better preparedness around these allows them to respond much faster to and leverage domestic and global events and trends. This unified structure enables management to take faster informed decisions with certainty, creating significant competitive leverage and unexpected benefits.

They indicated that it is important to have all communication filtered and coordinated through one Chief Internal Controls Officer (CICO) or an Internal Controls Committee (ICC). In their opinion, the CICO should be the person to ensure communication flow and that work is carried out in the manner required to reach objectives without problems. They note that this person/s should have the attributes of being a good communicator and understand how management, regulators, and controllers think. In this way, communication upwards and downwards flows faster and more 
efficiently, ensuring proactive actions. The methodology and structure of internal controls will differ depending on the size and objectives of the firm.

Moreover, they indicate that one of the most important functions carried out by their compliance, internal audit, and risk managers is the training of the line managers on controls. They are also the persons responsible to prepare policies and procedures together with the line managers, to guide them in their work. By involving management, a quick buy-in is obtained. This will also ensure that controls are not opposed and that help is given to identify risks and noncompliances/nonconformities. Compliance, internal audit, and risk management will continue to independently carry out their verifications, and however, communicate everything to the CICO or the ICC. The latter is in a better position to understand the business, since he/she has a birds-eye-view of the overall situation. She/he is better able to delegate internal audits and give appropriate recommendations in a proactive manner. However, participants also noted that the regulators had various unclear and conflicting views on this aspect. Since, although standards and regulations promote the responsibility of the Board of Directors to ensure appropriate governance and a mix of experience and academia, they indirectly promote individualism by ensuring segregation between the internal control functions and sometimes Chinese walls (as opposed to ensuring better communication), explaining that there is conflict of interests.

One of the participants brought up the findings of Calomiris and Haber (2014) in their book "Fragile by Design," which highlights one of the European countries - Malta - as having been crisis free and credit abundant (in the banking sectors) since the 1970. Similarly Singapore, Hong Kong, Canada, Australia, and New Zealand met these criteria. In the case of the three small "City states" or small Islands the reason was boiled down to a closed culture and, as the authors note, lack of diversity makes communication better and easier. Thus, indicating the importance of setting a similar culture and objective. Once this is ensured, the job of having the right governance structure and internal controls becomes a natural thing.

This thought was shared by (26) participants, who indicated that the focus should be that of ensuring the right culture to meet defined clear objectives and the way to arrive at this should be owned by the firm and not defined by the regulators as a "one-size-fits-all." This should be rebalanced on a periodic basis and whenever there are material changes in the objectives or risks arising from changes (both internal and external). Some have also indicated that policies should be flexible, allowing for 
quick changes. They have noted that sometimes they are too strict and sometimes too lax. Policy makers should ensure that they draw-up requirements that give default solutions but allow for exceptions, given they follow a certain procedure to obtain them. Besides, internal control should be adequate to avoid excessive bureaucracy, increased unnecessary approval times, etc.

\section{CONCLUSION}

In summary, derivatives are nowadays widely used globally and adequate internal control in a firm allows to diminish the risk of its misuse. The controls and those in charge of them have to look deeper into the firms and customize their governance and internal controls according to their objectives and determine a common culture. To do this it is essential to have all the information on transactions and improve the quality of communications internally between all units and departments and externally with the industry and the regulators. This cannot happen without improving the caliber of those effecting the control.

Although, the debate about control is still open, the interviewees believe that sound internal controls could avoid new debacles without adding other restrictions to the market. In the participants' opinion there is a need to change to a team culture eliminating the "us and them" mentality. Communication upwards and downwards would ideally be forthcoming and everyone is to ensure that the flow is easy, explained, and understood (especially between the Board of Directors and the internal control functions). Policies need to be "living documents" that change with time and innovations; are understandable and cater for the flexibility needed. Accordingly, it is advisable for firms to ensure that before entering into certain transactions, these are understood by the required persons. Ideally it is supported by regular training and enforced controls, wherein any nonconformities and actions taken to bring them back in line are documented and communicated to all (not only the internal controllers).

Therefore, as noted in this paper, when using derivatives, the designers of internal controls and governance need to be aware of derivatives design, the human aspect (internal and external culture, attributes required for people surrounding derivatives), regulations, accounting standards, communication (integration of the units), knowledge, and training within the firm. 


\section{REFERENCES}

Allayannis, G., Lel, U., \& Miller, D. P. (2012). The use of foreign currency derivatives, corporate governance, and firm value around the world. Journal of International Economics, $87(1), 65-79$.

Bezzina, F., \& Grima, S. (2012). Exploring factors affecting the proper use of derivatives: An empirical study with active users and controllers of derivatives. Managerial Finance, $38(4), 414-435$.

Braun, V., \& Clarke, V. (2006). Using thematic analysis in psychology. Qualitative Research in Psvchologv, 3(2), 77-101.

Breakwell, G. M., Hammond, S., \& Fife-Schaw, C. (Eds.). (1994). Research methods in psychology. London: Sage.

Calomiris, W. C., \& Haber, H. S. (2014). Fragile by design: The political origins of banking crisis \& scarce credit (pp. 454-455). Princeton University Press: Princeton, NJ.

Davison, B. (2000). Auditing derivative strategies. IIA Research Foundation: Altamonte Springs, FL.

Fernández-Laviada, A., Martínez-García, F. J., \& Montoya Del Corte, J. (2007). Internal control of derivatives usage by Spanish savings banks: An empirical survey. Spanish Accounting Review, 10(2), 127-164.

Géczy, C. C., Minton, B. A., \& Schrand, C. (2007). Taking a view: Corporate speculation, governance, and compensation. The Journal of Finance, 62(5), 2405-2443.

Global Derivatives Study Group. (1993). Derivatives: Practices and principles. Washington, DC: Group of Thirty.

Grima, S. (2011). A study of uses and misuses of derivatives. University of Malta, unpublished thesis (transcript of interview with Nick Leeson, 2009).

Guest, G., Bunce, A., \& Johnson, L. (2006). How many interviews are enough? An experiment with data saturation and variability. Field Methods, 18, 59-82.

Kang, R. (2007). Are derivatives for risk management or is risk management for derivatives. Retrieved from http://seekingalpha.com/article/35804-are-derivatives-for-risk-management-or-is-risk-management-for-derivatives. Accessed on January 2015.

Kinney, W., Maher, M., \& Wright, D. (1990). Assertions-based standards for integrated internal control. Accounting Horizons, 4, 1-8.

Patton, M. (1990). Qualitative evaluation and research methods. Newbury Park, CA: Sage.

Saunders, M., Lewis, P., \& Thornhill, A. (2003). Research methods for business students. London: Prentice Hall Financial Times.

The Committee of Sponsoring Organizations of the Treadway Commission (COSO). (1992). Internal control issues in derivatives usage. Retrieved from http://coso.org/documents/ Internal\%20Control\%20Issues\%20in\%20Derivatives\%20Usage.pdf. Accessed on July, 2015.

Zeidan, R., \& Müllner, J. (2015). Firm, market and top management antecedents of speculation: Lessons for corporate governance. Journal of Multinational Financial Management, $32-33,42-58$. 